\title{
What it takes to avoid equilibration
}

\author{
R. Gallego, ${ }^{1}$ H. Wilming, ${ }^{1}$ J. Eisert, ${ }^{1}$ and C. Gogolin ${ }^{2}$ \\ ${ }^{1}$ Dahlem Center for Complex Quantum Systems, Freie Universität Berlin, 14195 Berlin, Germany \\ ${ }^{2}$ ICFO-Institut de Ciencies Fotoniques, The Barcelona Institute of Science and Technology, 08860 Castelldefels (Barcelona), Spain
}

(Received 9 February 2018; revised manuscript received 13 June 2018; published 27 August 2018)

\begin{abstract}
Numerous works have shown that under mild assumptions, unitary dynamics inevitably leads to equilibration of physical expectation values if many energy eigenstates contribute to the initial state. Here, we consider systems driven by arbitrary time-dependent Hamiltonians as a protocol to prepare systems that do not equilibrate. We introduce a measure of the resilience against equilibration of such states, and we show, under natural assumptions, that in order to increase the resilience against equilibration of a given system, one needs to possess a resource system that itself has a large resilience. In this way, we establish a link between the theory of equilibration and resource theories by quantifying the resilience against equilibration and the resources that are needed to produce it. We connect these findings with insights into local quantum quenches, and we investigate the (im)possibility of formulating a second law of equilibration by studying how resilience can be either only redistributed among subsystems, if these remain completely uncorrelated, or in turn created in a catalytic process if subsystems are allowed to build up some correlations.
\end{abstract}

DOI: 10.1103/PhysRevA.98.022135

\section{INTRODUCTION}

When a complex quantum system is prepared in a pure state, it evolves in time under its Hamiltonian indefinitely and, after a sufficiently long passage of time, even returns to its initial state if it is finite-dimensional. Nevertheless, many physical observables quickly approach a stationary value and only rarely deviate significantly from this again. Over recent years, great progress has been made in understanding this equilibration phenomenon. Rigorous proof for such behavior under very general assumptions has been found [1-5], and at the same time it is the basis of a plethora of studies of quantum many-body systems out of equilibrium [6,7], motivated from a condensedmatter perspective.

A central quantity for proofs of equilibration in the context of pure-state quantum statistical mechanics is the so-called effective dimension. Roughly speaking, it measures with how many energy eigenstates a given initial state has a significant overlap. Provided that a system has a large effective dimension, one can prove under very mild additional assumptions-such as a nondegenerate energy gap condition-that expectation values of observables [1,3,8] and even whole subsystems [9] equilibrate (see Ref. [4] for a review). In addition, systems with a large effective dimension can be proven to equilibrate fast for certain observables with few outcomes [10], and systems fluctuate around equilibrium slowly [2], which forces weakly coupled systems to decohere [11]. We note, however, that it is an important open problem to prove general results that show how long it takes for a system to equilibrate, despite considerable recent progress on this question [5,12-18].

It has been argued that the effective dimension is large in realistic situations $[1,3,8,9]$. The core of such arguments is that, due to the exponential growth of the dimension of the Hilbert space of many-body systems, it is unreasonable to expect that an initial state will have overlap with only a few of them (except in gapped systems at very low temperature).
Indeed, if the system has a reasonably large energy uncertainty and its energy distribution is centered around some value (no strongly bimodal distribution), then one can argue that the effective dimension will grow exponentially with the system size, hence ensuring equilibration for large enough systems. While such arguments suggest quite convincingly that it may be difficult to bring a large many-body system to a state that would not equilibrate (and hence is not expected to happen spontaneously), they do not quantify this difficulty.

Here, we provide such a quantification by analyzing the question from a fresh perspective, namely that of the emergent field of quantum thermodynamics (see Refs. [19,20] for reviews), specifically that of resource theories, which is becoming increasingly important in that context. In this mind set, we consider the task of preparing states that can avoid equilibration when provided with another quantum system as a resource and allowing for a very general set of operations that includes arbitrary evolutions of both systems under arbitrary time-dependent Hamiltonians. This analysis, employing tools from resource theories of purity or coherence [20-27], leads to simple formulas expressing lower bounds on the amount of resources that are needed in order to prepare systems with a low effective dimension. Indeed, these bounds imply-given reasonable assumptions about the form of the resources- that it is difficult to bring large interacting many-body systems to a state that does not equilibrate, in the precise sense that it would require an amount of resources diverging with the system size. This provides stronger quantitative grounds to the arguments laid out above, suggesting that systems should realistically be expected to have a large effective dimension and thus equilibrate. We also connect these insights to the description of quantum quenches.

We then go further and study in a general fashion how the property of resisting equilibration behaves under the composition of systems. That is, we treat such a property as a 
quantifiable resource, and we investigate how it can be created or reshuffled among subsystems when one operates globally on them by making them interact and evolve under arbitrary time-dependent Hamiltonians. In particular, we show that it is possible to find certain systems, referred to as catalysts, which can be used to bring other systems to a state resisting equilibration while leaving the catalyst itself invariant. The implications of this insight are discussed in relation to what could be called a second law of equilibration, presenting additional challenges within the study of the equilibration properties of many-body systems.

This work is structured as follows: We first briefly review equilibration results in pure-state quantum statistical mechanics in Sec. II. In Sec. III, we present our framework to prepare states that do not equilibrate, and we provide general bounds for the resources required in the case of stationary initial states. We then study in Sec. IV how the resilience against equilibration can be either only redistributed among subsystems, if these remain completely uncorrelated, or in turn created in a catalytic process if subsystems are allowed to build up arbitrarily weak correlations. Finally, in Sec. V, we extend our results to the case of nonstationary states under additional assumptions about the process that brings the system out of equilibrium.

\section{EQUILIBRATION IN PURE-STATE QUANTUM STATISTICAL MECHANICS}

Let us summarize some of the important equilibration results $[1,3,4,9]$ to highlight the relevance of the effective dimension and set the notation. Consider a finite-dimensional quantum system evolving unitarily according to the Schrödinger equation through the states $\rho(t)$ starting from an initial state $\rho$ under a Hamiltonian $H$ with eigenvectors $\left\{\left|E_{k}\right\rangle\right\}_{k}$ and corresponding energies $\left\{E_{k}\right\}_{k}$. We assume that $H$ does not have any degenerate energy gaps, i.e., $H$ is nondegenerate and any energy difference between energy levels is unique. This condition is generically fulfilled for fully interacting Hamiltonians. For simplicity, we will henceforth assume that all appearing Hamiltonians have this property.

We denote by $\tau:=\lim _{T \rightarrow \infty} 1 / T \int_{0}^{T} d t$ the infinite timeaverage. Let $A$ be an arbitrary observable. Then its expectation value equilibrates to the expectation value in the dephased or time-averaged state $\omega_{H}(\rho):=\sum_{k} p_{k}\left|E_{k} \backslash E_{k}\right|=\overline{\rho(t)}$ with $p_{k}:=\left\langle E_{k}|\rho| E_{k}\right\rangle$ whenever the effective dimension

$$
d_{H}^{\text {eff }}(\rho):=\frac{1}{\sum_{k} p_{k}^{2}}=\frac{1}{\operatorname{Tr}\left[\omega_{H}(\rho)^{2}\right]}
$$

is sufficiently large. This is captured by the bound

$$
\operatorname{Var}_{H}(A, \rho):=\overline{\operatorname{Tr}\left(A\left[\rho(t)-\omega_{H}(\rho)\right]\right)^{2}} \leqslant \frac{\|A\|^{2}}{d_{H}^{\text {eff }}(\rho)} .
$$

A similar bound also holds in terms of the second largest eigenvalue of $\omega_{H}(\rho)$ instead of the effective dimension [1,3], but the formulation in terms of the effective dimension is more suitable for our purposes as it will allow us to state our results in terms of entropies [see Sec. VID for equilibration results in terms of the second largest eigenvalue of $\omega_{H}(\rho)$ instead of the effective dimension and a comparison to the effective dimension and von Neumann entropy of $\left.\omega_{H}(\rho)\right]$.
If the combined system is (mentally or physically) split into the tensor product of two subsystems $S$ and $B$, it can be shown that the whole state $\rho^{S}(t):=\operatorname{Tr}_{B}[\rho(t)]$ of the subsystem $S$ is, most of the time, close in trace distance to the local equilibrium state $\omega_{H}^{S}(\rho):=\operatorname{Tr}_{B}\left[\omega_{H}(\rho)\right]$ in the sense that [5,9]

$$
\overline{\mathcal{D}\left(\rho^{S}(t), \omega_{H}^{S}(\rho)\right)} \leqslant \frac{1}{2} \sqrt{\frac{d_{S}^{2}}{d_{H}^{\text {eff }}(\rho)}},
$$

where $d_{S}$ denotes the dimension of the Hilbert space of $S$. The trace distance measures the (single shot) distinguishability under arbitrary observables, so if the right-hand side of the above inequality is small, this means that for most $t$ there exists not a single observable that would enable one to tell apart $\rho^{S}(t)$ from $\omega_{H}^{S}(\rho)$. The above inequalities show that systems whose initial states have a large effective dimension appear to equilibrate to great precision, and it is hence natural to ask the following: How difficult is it to prepare a state of a quantum many-body system that can avoid equilibration?

\section{THE COST OF AVOIDING EQUILIBRATION}

Let us first, based on the role played by the effective dimension in the equilibration bounds (2) and (3), define the resilience (against equilibration) of a $d$-dimensional system initially in state $\rho$ evolving under the Hamiltonian $H$ as

$$
\mathcal{R}(\rho, H):=\log \left(\frac{d}{d_{H}^{\text {eff }}(\rho)}\right) .
$$

Having a high resilience is a necessary condition for avoiding equilibration in the long run. To further illustrate its relation to equilibration, consider, for example, $\rho(t)$ being the maximally mixed state for all $t$, which is, and stays, equilibrated under any Hamiltonian. In this case, $\mathcal{R}(\rho, H)=0$. The other extreme is given by a $\rho$ with $1<d_{H}^{\text {eff }}(\rho) \ll d$, which in general does not equilibrate. In this case, one obtains $\mathcal{R}(\rho, H) \approx \log d \propto$ $n$, where $n$ is the size (number of subsystems) of the whole system.

Note that a large resilience is a necessary, but not a sufficient, condition for a system to avoid equilibration, as can be seen by taking $\rho(t)$ to be an eigenstate of a Hamiltonian. For our work this is not problematic, as we will be concerned with obtaining lower bounds on the resources needed to prepare states with a large resilience. Our results show that states that do not equilibrate are difficult to prepare, whereas situations such as the case of $\rho(t)$ being an eigenstate only show that in addition some states that do equilibrate can also be difficult to prepare.

We now present a very general scenario that models possible preparations of quantum states out of equilibrium and which includes the common settings of quenches, ramps, and other control protocols. We consider a quantum system $Q$ that we want to bring out of equilibrium. For this, we make it interact arbitrarily with some other system $R$. The system $R$ may be physically interpreted as an environment into which $Q$ is placed as well as any precisely engineered resource employed with the purpose of bringing $Q$ out of equilibrium. More formally, we are given two quantum systems $Q$ and $R$ in the product state $\sigma^{Q} \otimes \sigma^{R}$ and with noninteracting Hamiltonian $H_{i}^{Q R}:=H_{i}^{Q} \otimes \mathbb{1}^{R}+\mathbb{1}^{Q} \otimes H_{i}^{R}$ (here we call a Hamiltonian 
noninteracting if and only if it is exactly of this form). For now, let us assume that both systems are stationary, i.e., $\left[\sigma^{Q} \otimes\right.$ $\left.\sigma^{R}, H_{i}^{Q R}\right]=0$. To bring $Q$ out of equilibrium, we are allowed to drive the evolution of the systems by changing the global Hamiltonian in a completely arbitrary fashion and making $Q$ and $R$ interact. More formally, we let the global system evolve unitarily under a time-dependent Hamiltonian $H^{Q R}(t)$ for $t \in\left[t_{i}, t_{f}\right]$ from the initial Hamiltonian $H^{Q R}\left(t_{i}\right)=H_{i}^{Q R}$ to the final Hamiltonian $H^{Q R}\left(t_{f}\right):=H_{f}^{Q R}=H_{f}^{Q} \otimes \mathbb{1}^{R}+\mathbb{1}^{Q} \otimes$ $H_{f}^{R}$, which must again be noninteracting. Given a pair of initial and final Hamiltonians, one can in principle always find a trajectory $\alpha$ that implements on the state any possible unitary $U_{\alpha}$. Even going beyond this set of operations, for the sake of generality, we allow the use of a source of randomness to implement a trajectory $\alpha$ between the two fixed Hamiltonians $H_{i}^{Q R}$ and $H_{f}^{Q R}$ with some probability $p_{\alpha}$. On average, the state on $Q R$ is then transformed as $\sigma^{Q R} \mapsto \sum_{\alpha} p_{\alpha} U_{\alpha} \sigma^{Q R} U_{\alpha}^{\dagger}$. All possible protocols bringing a system out of equilibrium in this way result in a unital map $\Lambda$ on the given quantum state, i.e., $\Lambda$ fulfills $\Lambda(\mathbb{1})=\mathbb{1}$. This is in fact the only property we need for our result below. To summarize, the process of bringing the system out of equilibrium can be described abstractly as

$$
\left(\sigma^{Q R}, H_{i}^{Q R}\right) \mapsto\left(\Lambda\left(\sigma^{Q R}\right), H_{f}^{Q R}\right)=:\left(\rho^{Q R}, H_{f}^{Q R}\right),
$$

with $\Lambda(\mathbb{1})=\mathbb{1}$.

Note that we allow for an unrealistically high degree of control over the precise trajectories of Hamiltonians, which only makes our bounds stronger, since we are interested in lower bounding the resources needed to prepare systems that do not equilibrate. Hence, incorporating fewer restrictions on the set of operations can only strengthen the applicability of our lower bounds. Nonetheless, we point out that incorporating further restrictions on the set of operations - such as finite energy input on the compound $Q R$ or limitations in controlmight provide tighter bounds of independent interest (see Sec. VII for a discussion).

Given the initial state and Hamiltonian and using the set of operations laid out above, the task is to optimally exploit the resource system $R$ by choosing an optimal final Hamiltonian and time-dependent trajectories, resulting in the channel $\Lambda$, to prepare a state on $Q$ so that it does not equilibrate for the chosen final Hamiltonian. Let us denote with $\rho_{t}^{Q}$ the time-evolved state of $Q$ under $H_{f}^{Q}$ after $\Lambda$ has been applied. Its initial condition is then

$$
\rho^{Q}=\operatorname{Tr}_{R}\left[\Lambda\left(\sigma^{Q} \otimes \sigma^{R}\right)\right] .
$$

To prepare $Q$ so that it does not equilibrate, one needs to obtain a sufficiently large resilience $\mathcal{R}\left(\rho^{Q}, H_{f}^{Q}\right)$. We are now in a position to formulate our first main result, which provides a general upper bound on that resilience in terms of the initial state and Hamiltonian.

Theorem 1. Resources for preparing resilient states from stationary ones. Consider a system $Q$ and resource $R$ given in the product state $\sigma^{Q} \otimes \sigma^{R}$ stationary with respect to the noninteracting Hamiltonian $H_{i}^{Q R}$. Then, for any final noninteracting Hamiltonian $H_{f}^{Q R}$ and operation of the form (6), the resulting state $\rho^{Q}$ fulfills

$$
\mathcal{R}\left(\rho^{Q}, H_{f}^{Q}\right)-\mathcal{R}\left(\sigma^{Q}, H_{i}^{Q}\right)=: \Delta \mathcal{R}^{Q} \leqslant \mathcal{R}\left(\sigma^{R}, H_{i}^{R}\right) .
$$

Before providing the proof of Theorem 1, let us discuss its interpretation and consequences. First note that the resulting resilience $\mathcal{R}\left(\rho^{Q}, H_{f}^{Q}\right)$ is upper-bounded by a function of the initial state and Hamiltonian only. If those initial conditions provide a small enough bound, then it follows that no protocol from the very general set of operations considered above can bring $Q$ from an equilibrating state to a state that does not equilibrate. Secondly, note that $\Delta \mathcal{R}^{Q}$ is the change of the resilience of $Q$. Hence, Eq. (7) states that the change in the resilience of $Q$ is upper-bounded by the resilience present in the resource $R$. This implies that the resilience against equilibration behaves like a "thermodynamic resource": in order to increase the resilience of $Q$ (so that it has a chance of avoiding equilibration), we need to possess another system $R$ from which to take this resilience. Indeed, in the particular case of not having any resource $R$, we see that (7) implies that the resilience is a monotone under unital maps. That is, it fulfills

$$
\mathcal{R}\left(\rho^{Q}, H_{f}^{Q}\right) \leqslant \mathcal{R}\left(\sigma^{Q}, H_{i}^{Q}\right) .
$$

While our bounds are phrased in a resource-theoretical language, it readily applies to paradigmatic situations from the context of quantum systems out of equilibrium such as quenches, ramps, and general control protocols, underlining its physical significance.

Corollary 2. Local quenches. Consider a many-body system $Q$ in an initial stationary state $\sigma^{Q}$ and initial Hamiltonian $H_{i}^{Q}$. Let $H_{f}^{Q}$ be any Hamiltonian on $Q$, and let $\Phi$ be any quantum channel (not necessarily unital) that acts nontrivially only on a subsystem $X$ of dimension $d_{X}$. Then

$$
\mathcal{R}\left(\Phi\left(\sigma^{Q}\right), H_{f}^{Q}\right)-\mathcal{R}\left(\sigma^{Q}, H_{i}^{Q}\right) \leqslant \log d_{X} .
$$

This statement implies that local quenches can alter the resilience only very little (see Sec. VIB for the proof of the corollary using a Stinespring dilation). A similar bound can be expected to hold if the Hamiltonian on $Q$ is only locally modified in a time-dependent manner for a finite time and with a bound on its norm, due to Lieb-Robinson bounds [28].

Let us consider now a further illustrating example of the general bound provided by Theorem 1. Let the state $\sigma^{Q}$ be a microcanonical state for the initial Hamiltonian $H_{i}^{Q}$, with an energy window containing $K(n)$ eigenstates. We allow the number of eigenstates to depend on the size $n$ of the system arbitrarily, but typically one expects $K(n)$ to grow approximately exponentially with $n$. Suppose that the resource consists of an $m$-partite system with local Hilbert space dimension $D$. In this case, one obtains

$$
d_{H_{f}^{Q}}^{\mathrm{eff}}\left(\rho^{Q}\right) \geqslant \frac{K(n)}{D^{m}}
$$

which implies that the size of the resource $m$ has to diverge if one wants to prepare a state that can avoid equilibration, as long as the number of states within the microcanonical window grows with $n$.

The proof of Theorem 1 is based on properties of Rényi divergences [29] (a similar proof can also be carried out using Tsallis entropies [30]). For any two quantum states $\sigma$ and $\tau$, 
the Rényi divergences are defined to be

$$
D_{\alpha}(\sigma \| \tau):=\frac{1}{1-\alpha} \log \left(\sigma^{\alpha} \tau^{1-\alpha}\right),
$$

with the corresponding Rényi- $\alpha$ entropies defined as $S_{\alpha}(\rho):=\log (d)-D_{\alpha}(\rho \| \mathbb{1} / d)$. The Rényi divergences for $\alpha \in[0,2]$ satisfy the data-processing inequality, i.e., for any quantum channel $\Phi$ it holds that

$$
D_{\alpha}(\Phi(\sigma) \| \Phi(\tau)) \leqslant D_{\alpha}(\sigma \| \tau)
$$

and they are additive on product states in the sense that

$$
D_{\alpha}\left(\sigma_{A} \otimes \sigma_{B} \| \tau_{A} \otimes \tau_{B}\right)=D_{\alpha}\left(\sigma_{A} \| \tau_{A}\right)+D_{\alpha}\left(\sigma_{B} \| \tau_{B}\right) .
$$

Combining Eqs. (1), (4), and (10), one can see that one can write the resilience in terms of the Rényi divergences of the dephased state as

$$
\mathcal{R}(\rho, H)=D_{2}\left(\omega_{H}(\rho) \| \mathbb{1} / d\right) .
$$

This quantity and variants thereof have been studied in the context of the resource theory of coherence and purity $[20,25]$. Indeed, $\mathcal{R}(\rho, H)$ can be understood as the purity of the timeaveraged state of $\rho$. Using additivity and the data-processing inequality, one can show the following two properties: First, for any state $\sigma$ that is stationary with respect to a Hamiltonian $H_{i}$, any unital channel $\Lambda$, and Hamiltonian $H_{f}$, one has that

$$
\mathcal{R}\left(\rho, H_{f}\right)=\mathcal{R}\left(\Lambda(\sigma), H_{f}\right) \leqslant \mathcal{R}\left(\sigma, H_{i}\right) .
$$

Second, consider product initial states $\sigma^{Q} \otimes \sigma^{R}$ and a noninteracting Hamiltonian $H_{i}^{Q R}$. If $\sigma^{Q}$ and $\sigma^{R}$ are stationary under the Hamiltonians $H_{i}^{Q}$ and $H_{i}^{R}$, respectively, we have that the resilience is additive,

$$
\mathcal{R}\left(\sigma^{Q} \otimes \sigma^{R}, H_{i}^{Q R}\right)=\mathcal{R}\left(\sigma^{Q}, H_{i}^{Q}\right)+\mathcal{R}\left(\sigma^{R}, H_{i}^{R}\right) .
$$

Given the properties (14) and (15), which are proven in Sec. VIA, the proof of Theorem 1 is straightforward:

Proof of Theorem 1. The concatenated map $\operatorname{Tr}_{R} \circ \Lambda$ is unital. Hence, using Eqs. (6) and (14), one obtains that

$$
\mathcal{R}\left(\rho^{Q}, H_{f}^{Q}\right) \leqslant \mathcal{R}\left(\sigma^{Q} \otimes \sigma^{R}, H_{i}^{Q R}\right) .
$$

In addition, $\sigma^{Q}$ and $\sigma^{R}$ are stationary states by assumption, hence one can use additivity (15), which yields

$$
\mathcal{R}\left(\rho^{Q}, H_{f}^{Q}\right) \leqslant \mathcal{R}\left(\sigma^{Q}, H_{i}^{Q}\right)+\mathcal{R}\left(\sigma^{R}, H_{i}^{R}\right) .
$$

At this point, it is worth commenting on the relation between our bounds and von Neumann entropy production. Note that the resilience can be expressed as a Rényi divergence (13), which is a measure of the purity of the dephased state $\omega_{H}(\rho)$. It is then natural to ask if similar results can be expressed in terms of the usual von Neumann entropy of the dephased state $S\left(\omega_{H}(\rho)\right)$. It is indeed the case that similar bounds to that of Theorem 1 can be easily proven for $S$. However, the quantity $S\left(\omega_{H}(\rho)\right)$ cannot be used to ensure equilibration in the spirit of Eqs. (2) and (3), hence bounds on the entropy cannot be argued to imply that the system avoids equilibration in the long run (see Theorem 3 and the discussion in Sec. VIE for details).

\section{THE SECOND LAW OF EQUILIBRATION AND CORRELATIONS}

As we have discussed after Theorem 1, the resilience against equilibration behaves like a thermodynamic resource in the sense that it cannot be created if it is not already present in another system. Nonetheless, (7) does not specify how much of the resource's resilience is spent in the process, it only quantifies how much must be present. We now turn to this question and show that it depends on the correlations created between $R$ and $Q$ in the process.

We consider again stationary given states $\sigma^{Q} \otimes \sigma^{R}$ as well as initial and final noninteracting Hamiltonians $H_{i}^{Q R}$ and $H_{f}^{Q R}$, respectively. We then implement an operation of the form (6), with the difference that we do not trace out the state of the resource, i.e., let

$$
\rho^{Q R}:=\Lambda\left(\sigma^{Q} \otimes \sigma^{R}\right)
$$

From the proof of Theorem 1, one obtains the bound

$$
\mathcal{R}\left(\rho^{Q R}, H_{f}^{Q R}\right) \leqslant \mathcal{R}\left(\sigma^{Q}, H_{i}^{Q}\right)+\mathcal{R}\left(\sigma^{R}, H_{i}^{R}\right) .
$$

If the resulting state is a product, $\rho^{Q R}=\rho^{Q} \otimes \rho^{R}$, one can show (see Lemma 6 in Sec. VIC) that

$$
\mathcal{R}\left(\rho^{Q}, H_{f}^{Q}\right)+\mathcal{R}\left(\rho^{R}, H_{f}^{R}\right) \leqslant \mathcal{R}\left(\rho^{Q} \otimes \rho^{R}, H_{f}^{Q R}\right),
$$

which implies in turn that $\Delta \mathcal{R}^{R}$, the change in the resilience of the resource $R$, satisfies

$$
-\Delta \mathcal{R}^{R}=\mathcal{R}\left(\sigma^{R}, H_{i}^{R}\right)-\mathcal{R}\left(\rho^{R}, H_{f}^{R}\right) \geqslant \Delta \mathcal{R}^{Q} .
$$

This inequality can be interpreted as a kind of second law of equilibration in the sense that to bring a system $Q$ from a stationary state into an initial state that can resist equilibration, another system $R$ must have been brought (closer) to an equilibrating state. In other words, the property of permanently remaining out of equilibrium, as measured by the resilience, can only be reshuffled between systems but not created. Note, however, that (21) relies on the assumption that the produced state $\rho^{Q R}$ is such that there are no correlations between $Q$ and $R$. This assumption may in many physically reasonable situations be unjustified. This leads us to the question of whether it is possible to derive a second law of equilibration that holds in full generality, or whether in contrast it is possible to make use of correlations between $Q$ and $R$ to violate (21). In the following theorem, we show that the latter is the case. Indeed, this is shown in the strongest possible form: $Q$ can be brought to a trajectory that does not equilibrate while $R$ remains unchanged and only a vanishing amount of correlations between $Q$ and $R$ need to be created for this. Furthermore, this is possible for arbitrarily large systems and even when the initial and final Hamiltonians are identical.

Theorem 3. No second law of equilibration. Consider a family of $n$-partite many-body systems with increasing $n$. There are stationary states $\sigma_{(n)}^{Q}$ and noninteracting initial and final Hamiltonians $H_{i,(n)}^{Q R}=H_{f,(n)}^{Q R}:=H_{(n)}^{Q R}$ such that for every $\epsilon>0$ there exist resource states $\sigma_{(n)}^{R}$ and channels of the form $\Lambda^{(n)}(\cdot)=\sum_{i} p_{i}^{(n)} U_{i}^{(n)} \cdot U_{i}^{(n)}$ with the following properties: 
(i) For all $n$, the resulting state

$$
\rho_{(n)}^{Q}=\operatorname{Tr}_{R}\left(\Lambda^{(n)}\left(\sigma_{(n)}^{Q} \otimes \sigma_{(n)}^{R}\right)\right)
$$

on $Q$ does not equilibrate under $H_{(n)}^{Q}$.

(ii) For all $n$, the state of the resource remains unchanged upon application of the channel: $\rho_{(n)}^{R}:=\operatorname{Tr}_{Q}\left(\Lambda^{(n)}\left(\sigma_{(n)}^{Q} \otimes \sigma_{(n)}^{R}\right)\right)=\sigma_{(n)}^{R}$.

(iii) For all $n$, the correlations between $R$ and $Q$ as measured by the mutual information remain arbitrarily small:

$$
I(Q: R):=D_{1}\left(\rho_{(n)}^{Q R} \| \rho_{(n)}^{Q} \otimes \rho_{(n)}^{R}\right) \leqslant \epsilon .
$$

(iv) The change $\Delta \mathcal{R}_{(n)}^{Q}$ of the resilience of the system diverges with $n \rightarrow \infty$.

The proof of Theorem 3 relies on a very recent result of Ref. [31], which establishes conditions on possible transitions within the resource theory of thermodynamics. All details, including a proof of Theorem 3 as well as the fact that the resilience of $\sigma_{(n)}^{R}$ diverges as $\epsilon \rightarrow 0$, are included in Sec. VIC.

Let us now discuss the implications of points (i)-(iii) of Theorem 3. We stress that these implications are independent of the particular measure for resilience against equilibration. As mentioned above, the theorem implies the existence of initial states $\sigma_{(n)}^{Q}$, which are perfectly equilibrated and can be brought to quantum states $\rho_{(n)}^{Q}$ that do not equilibrate, as expressed by property (i) while not spending any resources. It is important to highlight here that we consider the strongest possible notion of not equilibrating: In the proof of Theorem 3 we construct an observable $A^{(n)}$ of fixed norm, so that the fluctuations in time as measured by $\operatorname{Var}_{H_{(n)}^{Q}}\left(A^{(n)}, \rho_{(n)}^{Q}\right)$ are independent of the size $n$. That is, one could in principle measure this observable and physically observe the fluctuations for arbitrarily large systems. This, by property (ii), can be done while the state of $R$ remains unchanged.

Importantly, we highlight that we provide a very physical example of states $\sigma_{(n)}^{Q}$ with the above properties. Indeed, $\sigma_{(n)}^{Q}$ can be chosen to be the microcanonical ensemble of any possible Hamiltonian (see Sec. VIC), which are physical states that appear ubiquitously as a description of many-body systems at equilibrium.

Even more surprisingly, as $R$ does not change in the process, it is possible to iterate this procedure with a sequence of many subsystems $Q_{1}, \ldots, Q_{m}$ by reusing each time the same resource $R$, which is thus independent of $m$. This is possible as long as the different systems $Q_{1}, \ldots, Q_{m}$ do not interact with each other, and it brings each of the arbitrarily many subsystems to a state that does not equilibrate. More precisely, the theorem has the surprising implication that given the right resource $\sigma^{R}$ and an environment in the microcanonical statecomposed of an arbitrarily large number of $m$ noninteracting subsystems - one can, without spending any of that resource, turn the entire environment into a state that is (and remains) nowhere in equilibrium. Note that this is in sharp contrast with the situation covered by Theorem 4 concerning fully interacting systems, for which the resources necessary to avoid equilibration diverge with the system size. This highlights and clarifies the importance of interactions, even arbitrarily small ones, for equilibration. The extreme contrast between the noninteracting and the arbitrarily weakly interacting case is ultimately a result of the fact that an arbitrarily small weak interaction can lead to equilibration after a sufficiently long time. It is important to stress that by noninteracting systems we refer to completely uncoupled systems and not quasifree fermionic or bosonic systems.

Let us now relate Theorem 3 to the second-law-like inequality (21) to better understand the influence of correlations. First note that in full generality, even if $R$ and $Q$ are correlated, one can use Eqs. (14) and (15) to derive the condition (19), which can be straightforwardly rewritten as

$$
-\Delta \mathcal{R}^{R} \geqslant \Delta \mathcal{R}^{Q}+\mathcal{C}\left(\rho^{Q R}, H^{Q R}\right),
$$

where the term $\mathcal{C}\left(\rho^{Q R}, H^{Q R}\right):=\mathcal{R}\left(\rho^{Q R}, H^{Q R}\right)-$ $\mathcal{R}\left(\rho^{Q}, H^{Q}\right)-\mathcal{R}\left(\rho^{R}, H^{R}\right)$ provides the correction to $(21)$ due to correlations. Then, a consequence of property (iv) is that $\mathcal{C}\left(\rho^{Q R}, H^{Q R}\right)$ can be made negative and arbitrarily large in absolute value, even if the correlations are arbitrarily small as measured in mutual information, as shown by property (iii). The negativity of $\mathcal{C}\left(\rho^{Q R}, H^{Q R}\right)$ captures that $\mathcal{R}$ does not fulfill superadditivity, which is ultimately a consequence of the Rényi-2 entropy not fulfilling subadditivity [32].

Interestingly, these implications of Theorem 3 for the lack of superadditivity of $\mathcal{R}$ can be extended to alternative definitions of the resilience, some of which are based on entropies other than the Rényi-2 entropy. In Sec. VID we discuss alternative definitions of resilience and possible improvements of equilibration bounds, and in Sec. VIE we discuss the relation between Theorem 3 and subadditivity. Further, we show that it is impossible to construct bounds of the form of Eqs. (2) and (3) in terms of the Rényi entropies with $\alpha \leqslant 1$, which also excludes the usual von Neumann entropy corresponding to $\alpha=1$. Since the known bounds (2) and (3) are formulated in terms of $\alpha=2$ [recall that $d_{H}^{\text {eff }}(\rho)=e^{S_{2}\left(\omega_{H}(\rho)\right)}$ ], this only leaves open the possibility to derive improved versions of (2) and (3) in terms of Rényi entropies with $1<\alpha<2$, none of which could lead to a subadditive notion of resilience. Importantly, however, bounds expressed in terms of Rényi entropies with $1<\alpha<2$ would exhibit the same scaling behavior as our bounds due to the inequality [33]

$$
S_{\alpha}(\rho) \geqslant S_{\infty}(\rho) \geqslant \frac{\alpha-1}{\alpha} S_{\alpha}(\rho),
$$

which holds for any $\alpha>1$.

\section{NONSTATIONARY STATES}

In the previous sections, we considered the case in which the given states $\sigma^{Q}$ and $\sigma^{R}$ are stationary, which is arguably the most natural one in the context considered. One is given a system that is always perfectly equilibrated and shall use resources to prepare it such that it can avoid equilibration. Nonetheless, it is also possible to incorporate nonstationary states into our formalism by including an extra assumption on the set of operations. To motivate this assumption, let us first consider a simple example without resource system $R$. Consider only the system $Q$ provided in a pure state $\sigma^{Q}$ and Hamiltonian $H_{i}^{Q}$ so that $\sigma^{Q}=\left|\psi^{Q}\right\rangle\left\langle\psi^{Q}\right|$ with

$$
\left|\psi^{Q}\right\rangle=\frac{1}{\sqrt{d_{Q}}} \sum_{k}\left|E_{k}\right\rangle,
$$


where $d_{Q}$ is the Hilbert space dimension of $Q$. This state is highly nonstationary, but it has a null resilience against equilibration. It is further easy to construct a unitary evolution $U$ so that $\rho^{Q}=\Lambda\left(\sigma^{Q}\right)=U \sigma^{Q} U^{\dagger}$ has a resilience proportional to $d_{Q}$. This can be done by simply applying a unitary rotation that leaves the state in a superposition of two eigenstates of the chosen final Hamiltonian $H_{f}^{Q}$. This shows that preparing states that do not equilibrate from states such as $\sigma^{Q}$, which are not stationary, is perfectly possible within our set of operations, even without employing any resource state $R$. Note, however, that since $\sigma^{Q}$ is not stationary, applying the same unitary $U$ at a slightly different time $T$ will in general lead to a very different state $\Lambda \circ \mathcal{T}_{T}^{H_{i}{ }^{Q}}\left(\sigma^{Q}\right) \neq \rho^{Q}$, where we introduced the time-evolution operator

$$
\mathcal{T}_{t}^{H}(\cdot):=e^{-i H t} \cdot e^{i H t} .
$$

Hence, the subsequent time evolution cannot repeatedly be prepared unless one has unrealistically fine control over the time $T$ which determines when we initiate the time-dependent evolution on the system. This is crucial if one wants to reproduce many instances of the experiment in order to gather statistics and verify the nonequilibrating dynamics.

Therefore, it is interesting to consider unital preparation procedures $\Lambda$ that do not require such fine control over $T$. More precisely, and now considering the problem in more generality by including a system $R$, the extra assumption on the set of operations is that for every $t$ there exists a $t^{\prime}$ so that

$$
\Lambda \circ \mathcal{T}_{t}^{H_{i}^{Q R}}\left(\sigma^{Q} \otimes \sigma^{R}\right)=\mathcal{T}_{t^{\prime}}^{H_{f}^{Q R}} \circ \Lambda\left(\sigma^{Q} \otimes \sigma^{R}\right)
$$

[note that this contains formally as a particular case the situation of stationary states $\sigma^{Q} \otimes \sigma^{R}$ for which (28) is fulfilled taking $\left.t^{\prime}=0\right]$. Further note that we can also allow here for a family of unital maps $\left\{\Lambda_{s}\right\}$, possibly depending on some time $s>0$, each of which fulfills (28).

To analyze the condition (28), let us first consider the simpler case of $H_{i}^{Q R}=H_{f}^{Q R}:=H$. There exist at least two well-known classes of operations that fulfill the condition in this case. The first class is given by the so-called covariant maps defined as the maps that fulfill $\Lambda_{s} \circ \mathcal{T}_{t}^{H}=\mathcal{T}_{t}^{H} \circ \Lambda_{s}$ for all $t, s>0$. Such covariant maps can be motivated from different perspectives. Argued from the perspective of resource theories, they correspond to the set of operations that can be performed without a reference frame for time (see Ref. [34] and references therein) and relate to physically relevant scenarios such as Hamiltonian evolution under the rotating-wave approximation [35]. We also note that a similar condition appears in the context of fluctuation relations in the resource theory of thermodynamics [36]. An important example from the theory of many-body nonequilibrium dynamics is constituted by Markovian dynamics reflected by a dynamical semigroup $s \mapsto \Lambda_{s}(\rho)=e^{\mathcal{L} s}(\rho)$ with the property that $\mathcal{L}([H, \cdot])=[H, \mathcal{L}(\cdot)]$, corresponding to some dissipative dynamics. In the case $H_{i}^{Q R} \neq H_{f}^{Q R}$, the condition generalizes to $\mathcal{L}\left(\left[H_{i}^{Q R}, \cdot\right]\right)=\left[H_{f}^{Q R}, \mathcal{L}(\cdot)\right]$. It is important to stress, however, that (28) is substantially weaker than full covariance as it must only be fulfilled for the particular state $\sigma^{Q} \otimes \sigma^{R}$ and allows for $t \neq t^{\prime}$.
The second class is given by operations fulfilling $\Lambda=$ $\Lambda^{\prime} \circ \omega_{H_{i}^{Q R}}$, which in turn implies that $\Lambda \circ \mathcal{T}_{t}^{H_{i}^{Q R}}=\Lambda$. Such operations first put the system into a fully time-averaged state and then act on that.

For nonstationary provided states $\sigma^{Q R}$, in addition to assumption (28), we need to put an assumption on the Hamiltonians. Namely, we impose the condition

$$
\omega_{H_{i}^{Q R}}=\omega_{H_{i}^{Q}} \otimes \omega_{H_{i}^{R}}
$$

which is generically fulfilled and simply implies that letting the two noninteracting and initially uncorrelated systems $Q$ and $R$ equilibrate does not generate correlations between equilibrating observables. For example, it follows that if there are no distinct eigenvalues, $E_{k}^{Q} \neq E_{l}^{Q}$ of $H_{i}^{Q}$ and $E_{m}^{R} \neq E_{n}^{R}$ of $H_{i}^{R}$ so that $E_{k}^{Q}-E_{l}^{Q}=E_{m}^{R}-E_{n}^{R}{ }^{1}{ }^{1}$ We are now ready to formulate a generalization of Theorem 1 to nonstationary $\sigma^{Q}, \sigma^{R}$ for unital maps $\Lambda$ that fulfill condition (28).

Theorem 4. Resources needed to prepare resilient states. Consider a system $Q$ and resource $R$ provided in the product state $\sigma^{Q} \otimes \sigma^{R}$ and with Hamiltonian $H_{i}^{Q R}$ fulfilling $\omega_{H_{i}^{Q R}}=$ $\omega_{H_{i}{ }^{Q}} \otimes \omega_{H_{i}^{R}}$. Then, for any final noninteracting Hamiltonian $H_{f}^{Q R}$ and operation of the form (6) with $\Lambda$ fulfilling Eq. (28), the resulting state $\rho^{Q}$ fulfills

$$
\mathcal{R}\left(\rho^{Q}, H_{f}^{Q}\right)-\mathcal{R}\left(\sigma^{Q}, H_{i}^{Q}\right)=: \Delta \mathcal{R}^{Q} \leqslant \mathcal{R}\left(\sigma^{R}, H_{i}^{R}\right) .
$$

In the proof, one uses Eq. (28) to show that for such channels, the monotonicity relation Eq. (14) is also true for nonstationary states. Using the property $\omega_{H_{i}{ }^{Q R}}=\omega_{H_{i}{ }^{Q}} \otimes \omega_{H_{i}^{R}}$, one shows that the additivity relation Eq. (15) is also fulfilled for nonstationary states $\sigma^{Q R}$ (see Sec. VIA). The rest of the proof is then completely analogous to that of Theorem 1.

\section{TECHNICAL RESULTS AND PROOFS}

\section{A. Monotonicity and additivity of the resilience}

Let us first show the monotonicity of the resilience [Eq. (14)]. For the proof of Theorem 1, one requires monotonicity of the resilience for unital channels $\Lambda$ and stationary given states $\sigma^{Q}$ and $\sigma^{R}$, as expressed before Eq. (14). For Theorem 4 , one requires monotonicity for nonstationary given states $\sigma^{Q R}$, but it is sufficient to prove it for unital channels $\Lambda$, which fulfill in addition

$$
\Lambda \circ \mathcal{T}_{t}^{H_{i}^{Q R}}\left(\sigma^{Q} \otimes \sigma^{R}\right)=\mathcal{T}_{t^{\prime}}^{H_{f}^{Q R}} \circ \Lambda\left(\sigma^{Q} \otimes \sigma^{R}\right) .
$$

Note that this condition is automatically fulfilled for any $\Lambda$ whenever $\sigma^{Q R}$ is stationary. Hence, it suffices to show monotonicity of the resilience for maps fulfilling (31). In the following, we drop the superscripts $Q R$ when not explicitly needed to simplify the notation.

Because of the definition of $\omega_{H}$ [Eq. (1)], for any two fixed Hamiltonians $H_{i}$ and $H_{f}$, we can employ condition (31) to

\footnotetext{
${ }^{1}$ We assumed that for the results on equilibration to hold, $H^{Q}$ and $H^{R}$ have no degeneracies. Hence, this condition implies that $H^{Q R}$ has also no degeneracies. But Eq. (15) actually still holds even if both $H^{Q}$ and $H^{R}$ have degeneracies, as long as adding them does not create additional ones.
} 
show in a compact way that

$$
\begin{aligned}
& \left(\omega_{H_{f}} \circ \Lambda \circ \omega_{H_{i}}\right)(\sigma) \\
& =\left(\omega_{H_{f}} \circ \Lambda \circ \lim _{T \rightarrow \infty} \frac{1}{T} \int_{0}^{T} d t \mathcal{T}_{t}^{H_{i}}\right)(\sigma) \\
& =\lim _{T \rightarrow \infty} \frac{1}{T} \int_{0}^{T} d t\left(\omega_{H_{f}} \circ \Lambda \circ \mathcal{T}_{t}^{H_{i}}\right)(\sigma) \\
& =\lim _{T \rightarrow \infty} \frac{1}{T} \int_{0}^{T} d t\left(\omega_{H_{f}} \circ \mathcal{T}_{t^{\prime}}^{H_{f}} \circ \Lambda\right)(\sigma) \\
& =\lim _{T \rightarrow \infty} \frac{1}{T} \int_{0}^{T} d t\left(\omega_{H_{f}} \circ \Lambda\right)(\sigma) \\
& =\left(\omega_{H_{f}} \circ \Lambda\right)(\sigma),
\end{aligned}
$$

where (34) follows from condition (31), (35) follows from the fact that $\omega_{H} \circ \mathcal{T}_{t}^{H}=\omega_{H}$ for all $t>0$, and (36) follows simply because the integrand does not depend on $t$. With this we can now show monotonicity as

$$
\begin{aligned}
R\left(\sigma, H_{i}\right)= & D_{2}\left(\omega_{H_{i}}(\sigma) \| \mathbb{1} / d\right) \\
= & D_{2}\left(\omega_{H_{i}}(\sigma) \| \omega_{H_{i}}(\mathbb{1} / d)\right) \\
\geqslant & D_{2}\left(\left(\omega_{H_{f}} \circ \Lambda \circ \omega_{H_{i}}\right)(\sigma) \|\right. \\
& \left.\times\left(\omega_{H_{f}} \circ \Lambda \circ \omega_{H_{i}}\right)(\mathbb{1} / d)\right) \\
= & D_{2}\left(\left(\omega_{H_{f}} \circ \Lambda\right)(\sigma) \| \mathbb{1} / d\right) \\
= & R\left(\Lambda(\sigma), H_{f}\right),
\end{aligned}
$$

where (38) follows since $\omega_{H}$ is a unital map, (39) is a consequence of the data-processing inequality (11), and (40) follows from (36) and the fact that $\Lambda$ is a unital map.

We now turn to prove additivity of the resilience (15). For the proof of Theorem 1, one requires additivity of the resilience for given states $\sigma^{Q R}=\sigma^{Q} \otimes \sigma^{R}$ that are stationary with respect to a noninteracting Hamiltonian $H_{i}^{Q R}$. In this case, one finds

$$
\begin{aligned}
\omega_{H_{i}^{Q R}}\left(\sigma^{Q R}\right) & =\omega_{H_{i}^{Q R}}\left(\sigma^{Q} \otimes \sigma^{R}\right) \\
& =\omega_{H_{i}^{Q}}\left(\sigma^{Q}\right) \otimes \omega_{H_{i}^{R}}\left(\sigma^{R}\right) .
\end{aligned}
$$

For the proof of Theorem 4, one needs to show additivity for nonstationary initial states under the extra assumption that

$$
\omega_{H_{i}^{Q R}}=\omega_{H_{i}^{Q}} \otimes \omega_{H_{i}^{R}},
$$

which in turn implies (43). Hence, we conclude that Eq. (43) is fulfilled under the conditions of both Theorems 1 and 4 . We can now use this equation together with additivity of the Rényi divergences to show additivity of the resilience as

$$
\begin{aligned}
\mathcal{R}\left(\sigma^{Q} \otimes \sigma^{R}, H_{i}^{Q R}\right)= & D_{2}\left(\omega_{H_{i}^{Q R}}\left(\sigma^{Q} \otimes \sigma^{R}\right) \| \mathbb{1} / d_{Q R}\right) \\
= & D_{2}\left(\omega_{H_{i}^{Q}}\left(\sigma^{Q}\right) \otimes \omega_{H_{i}^{R}}\left(\sigma^{R}\right) \| \mathbb{1} / d_{Q R}\right) \\
= & D_{2}\left(\omega_{H_{i}^{Q}}\left(\sigma^{Q}\right) \| \mathbb{1} / d_{Q}\right) \\
& +D_{2}\left(\omega_{H_{i}^{R}}\left(\sigma^{R}\right) \| \mathbb{1} / d_{R}\right) \\
= & \mathcal{R}\left(\sigma^{Q}, H_{i}^{Q}\right)+\mathcal{R}\left(\sigma^{R}, H_{i}^{R}\right)
\end{aligned}
$$

\section{B. Local quenches}

The statement of Corollary 2 is an immediate consequence of Theorem 1. Consider a quantum many-body system initially prepared in $\sigma^{Q}$. Then one applies the local quantum channel $\Phi$ to a subsystem $X$ of $Q$ that is $d_{X}$-dimensional. To implement this quantum channel, one considers an auxiliary system $R$ of dimension $d_{R}=d_{X}$ initially prepared in a mixed state $\sigma^{R}$ so that

$$
\Phi\left(\sigma^{Q}\right)=\operatorname{Tr}_{R}\left(U\left(\sigma^{Q} \otimes \sigma^{R}\right) U^{\dagger}\right)
$$

for a suitable unitary $U$ acting upon $R$ and $Q$. Such a state $\sigma^{R}$ and unitary $U$ exist for any quantum channel $\Phi$ [32]. Since we can choose the Hamiltonian on $R$ freely, we can assume that $\sigma^{R}$ is stationary. The statement of the corollary then follows from Theorem 1 and the bound

$$
\mathcal{R}\left(\sigma^{R}, H_{i}^{R}\right) \leqslant \log \left(d_{R}\right)=\log \left(d_{X}\right),
$$

which immediately follows from the definition of the effective dimension.

\section{The role of correlations}

In this subsection, we discuss in more detail the role of correlations and the proof of Theorem 3 . We begin with the proof of Theorem 3. As stated earlier, it relies on the following theorem, which we adapt from Ref. [31], whereby for any state $\sigma$ we denote by $S(\sigma)$ its von Neumann entropy.

Theorem 5 (Ref. [31]). For any two finite-dimensional states $\sigma^{Q}$ and $\rho^{Q}$ of the same dimension with $S\left(\sigma^{Q}\right) \leqslant S\left(\rho^{Q}\right)$, for any $\delta I>0$, and for any $\epsilon>0$ there exist a finite-dimensional state $\sigma^{R}$ and a mixture of unitaries $\Lambda$, which yield the following:

(a) The channel produces the state $\rho^{Q}$ on $Q$ to accuracy $\epsilon>0$,

$$
\left\|\rho^{Q}-\operatorname{Tr}_{R}\left(\Lambda\left(\sigma^{Q} \otimes \sigma^{R}\right)\right)\right\|_{1}<\epsilon .
$$

(b) The state on $R$ after $\Lambda$ coincides with the state in which it was originally given:

$$
\sigma^{R}=\operatorname{Tr}_{Q}\left(\Lambda\left(\sigma^{Q} \otimes \sigma^{R}\right)\right)=: \rho^{R} .
$$

(c) The mutual information between $R$ and $Q$ after $\Lambda$ has acted is upper-bounded by $\delta I$,

$$
D_{1}\left(\Lambda\left(\sigma^{Q} \otimes \sigma^{R}\right) \| \rho^{Q} \otimes \rho^{R}\right) \leqslant \delta I .
$$

The Hilbert space dimension of $R$ may in general depend on both $\epsilon$ and $\delta I$.

Proof of Theorem 3. For any fixed finite-dimensional Hilbert space, the above Theorem 5 shows that whenever a state $\rho^{Q}$ has higher von Neumann entropy than the state $\sigma^{Q}$, then properties (ii) and (iii) of Theorem 3 are met. It remains to be shown that it is always possible to find states $\sigma^{Q}$ and $\rho^{Q}$ that simultaneously fulfill $S\left(\sigma^{Q}\right) \leqslant S\left(\rho^{Q}\right)$, have property (iv) of Theorem 3, and are such that $\rho^{Q}$ does not equilibrate [property (i)].

As required, we will now show that there exist $\sigma_{(n)}^{Q}$ and $\rho_{(n)}^{Q}$ with the following properties:
(i) $S\left(\sigma_{(n)}^{Q}\right) \leqslant S\left(\rho_{(n)}^{Q}\right)$,
(ii) $\Delta \mathcal{R}_{(n)}^{Q} \rightarrow \infty$ as $n \rightarrow \infty$,
(iii) $\rho_{(n)}^{Q}$ does not equilibrate. 
In the following, let $d=D^{n}$ be the dimension of the Hilbert space of the $n$-partite system. The initial states $\rho_{(n)}^{Q}$ of the nonequilibrating trajectories can be taken to be of the form

$$
\rho_{(n)}^{Q}=a|\Psi\rangle\langle\Psi|+(1-a) \frac{\Pi}{d-2},
$$

where $|\Psi\rangle=\left(\left|E_{1}\right\rangle+\left|E_{2}\right\rangle\right) / \sqrt{2}$ is a superposition of two arbitrary energy eigenstates of the Hamiltonian $H_{(n)}^{Q}$ (which can be chosen arbitrarily), $\Pi$ is the projector on the subspace orthogonal to $\left|E_{1}\right\rangle$ and $\left|E_{2}\right\rangle$, and $a \in[0,1]$. This family of states has an entropy given by

$$
\begin{aligned}
S_{1}\left(\rho_{(n)}^{Q}\right)= & a S_{1}(|\Psi \backslash X \Psi|)+(1-a) S_{1}\left(\frac{\Pi}{d-2}\right)+H_{2}(a), \\
= & (1-a) n \log (D) \\
& +(1-a) \log \left(1-2 D^{-n}\right)+H_{2}(a), \\
\approx & (1-a) n \log (D)+H_{2}(a),
\end{aligned}
$$

where $H_{2}(a)=-a \log (a)-(1-a) \log (1-a)$ is the binary entropy of $a$, and the error in the last approximation is exponentially small in $n$ for large $n$. On the other hand, the effective dimension of $\rho_{(n)}^{Q}$ approaches a constant:

$$
d_{H_{(n)}^{Q}}^{\mathrm{eff}}\left(\rho_{(n)}^{Q}\right)=\frac{1}{a^{2}+\frac{(1-a)^{2}}{d-2}} \leqslant \frac{1}{a^{2}} .
$$

In Sec. VID, this scaling is illustrated in more detail. It is clear that the states $\rho_{(n)}^{Q}$ do not equilibrate since there will be Rabi oscillations at frequency $E_{1}-E_{2}$ with amplitude $a$ independent of $n$ for all times. This proves condition (iii).

We now turn to constructing the family of given states $\sigma_{(n)}^{Q}$. Clearly, for any finite $n$ such states can be constructed. We now focus, therefore, on the case of arbitrarily large $n$. To fulfill condition (i) for large enough $n$, it suffices to have

$$
S_{1}\left(\sigma_{(n)}^{Q}\right) \leqslant(1-\tilde{a}) n \log (D)
$$

for any $\tilde{a}>a$. To fulfill condition (ii), it suffices to have

$$
\lim _{n \rightarrow \infty} S_{2}\left(\sigma_{(n)}^{Q}\right)=\infty .
$$

To see this, note that the resilience of the initial state can be written as

$$
\mathcal{R}\left(\sigma_{(n)}^{Q}, H_{(n)}^{Q}\right)=\log (d)-S_{2}\left(\sigma_{(n)}^{Q}\right),
$$

which holds for any Hamiltonian $H_{(n)}^{Q}$ for which $\sigma_{(n)}^{Q}$ is stationary. Using this together with the bound on the effective dimension of the final state (58), we obtain for the change of resilience

$$
\begin{aligned}
\Delta \mathcal{R}^{Q} & =\mathcal{R}\left(\rho_{(n)}^{Q}, H_{(n)}^{Q}\right)-\mathcal{R}\left(\sigma_{(n)}^{Q}, H_{(n)}^{Q}\right) \\
& \geqslant \log \left(\frac{d}{a^{2}}\right)-\mathcal{R}\left(\sigma_{(n)}^{Q}, H_{(n)}^{Q}\right) \\
& =S_{2}\left(\sigma_{(n)}^{Q}\right)-2 \log (a) .
\end{aligned}
$$

Hence we see that the change in resilience becomes arbitrarily large as long as the Rényi-2 entropy of the given states on $Q$ diverges with $n$. In general, the Rényi-2 entropy $S_{2}$ is upper bounded by the von Neumann entropy $S_{1}$, but it can be arbitrarily close to the latter. Therefore, a diverging $S_{2}$ is well compatible with Eq. (59).

We will now provide examples of states that indeed fulfill Eqs. (59) and (60), which concludes the proof. There exist in fact many families of states fulfilling those conditions, but we present as an example a family of microcanonical states $\sigma_{\Pi}^{(n)}$, which is maximally mixed on a subspace $\Pi$ of dimension $d^{\gamma}$ with $\gamma<1$. This is the scaling that one expects for an actual microcanonical state in a local many-body system, since it leads to an entropy scaling extensively with the system size:

$$
S_{1}\left(\sigma_{\Pi}^{(n)}\right)=\gamma n \log (D) .
$$

The constant $\gamma>0$ will of course depend on the effective temperature of the state (i.e., the temperature of the canonical state with the same mean energy) and it can be made arbitrarily small. Lastly, note that the Rényi-2 entropy diverges for any value of $\gamma>0$,

$$
\begin{aligned}
S_{2}\left(\sigma_{\Pi}^{(n)}\right) & =-\log \operatorname{Tr}\left[\left(\sigma_{\Pi}^{(n)}\right)^{2}\right] \\
& =-\log d^{-\gamma}=\gamma n \log (D) .
\end{aligned}
$$

This completes the proof.

Another natural example of states fulfilling (59) and (60) is given by stationary initial states that fulfill exponential clustering of correlations and have nonmaximal entropy density. Indeed, it has been proven recently that states with exponential decay of correlations have diverging effective dimension [37] with respect to local Hamiltonians.

Finally, as a side note, let us show that the resilience in the state of the resource $\mathcal{R}\left(\sigma_{(n)}^{R}, H_{(n)}^{R}\right)$, and hence also its Hilbert space dimension, must diverge as $\epsilon \rightarrow 0$. Suppose on the contrary that this would not be true. Then there would exist constants $C$ such that

$$
\mathcal{R}\left(\sigma_{(n)}^{R}, H_{(n)}^{R}\right) \leqslant C_{n}, \quad \forall \epsilon .
$$

For simplicity, we now fix a system size and drop all the $n$-dependence. We can use the resource $\sigma^{R}$ to bring $m$ uncorrelated copies of $\sigma^{Q}$ into a nonequilibrating state $\rho^{Q_{1} \cdots Q_{m}}$. By combining Theorem 1 with the bound (68), we would then obtain

$$
C \geqslant \mathcal{R}\left(\rho^{Q_{1} \cdots Q_{m}}, H^{Q_{1} \cdots Q_{m}}\right)-m \mathcal{R}\left(\sigma^{Q_{1}}, H^{Q_{1}}\right) .
$$

Since in each step we only correlated one of the systems with $R$ by an amount at most $\epsilon$, the final mutual correlations between the different copies of $Q$ are also bounded by $\epsilon: I\left(Q_{i}: Q_{j}\right) \leqslant$ $\epsilon$. Furthermore, the above equation holds, by assumption, for all $\epsilon$. We can then take the limit $\epsilon \rightarrow 0$. In this limit, we have

$$
\lim _{\epsilon \rightarrow 0} \rho^{Q_{1} \cdots Q_{m}}=\rho^{Q_{1}} \otimes \cdots \otimes \rho^{Q_{m}} .
$$

Since the resilience is superadditive on product states, as we show in Lemma 6 below, we would therefore obtain

$$
C \geqslant m\left(\mathcal{R}\left(\rho^{Q_{1}}, H^{Q_{1}}\right)-\mathcal{R}\left(\sigma^{Q_{1}}, H^{Q_{1}}\right)\right) .
$$

Since this equation holds for all $m$, we obtain a contradiction.

Lemma 6. Superadditivity on product states. The resilience is superadditive on product states of noninteracting 
systems:

$$
\mathcal{R}\left(\rho^{Q} \otimes \rho^{R}, H^{Q R}\right) \geqslant \mathcal{R}\left(\rho^{Q}, H^{Q}\right)+\mathcal{R}\left(\rho^{R}, H^{R}\right)
$$

if $H^{Q R}=H^{Q} \otimes \mathbb{1}+\mathbb{1} \otimes H^{R}$.

Proof. By using the integral representation of $\omega_{H}$ as a timeaverage, it follows immediately that local and global dephasing commutes. We thus have

$$
\begin{aligned}
\omega_{H^{Q}} \otimes \omega_{H^{R}} & =\omega_{H^{Q R}} \circ\left(\omega_{H^{Q}} \otimes \omega_{H^{R}}\right) \\
& =\left(\omega_{H} Q \otimes \omega_{H^{R}}\right) \circ \omega_{H^{Q R}} .
\end{aligned}
$$

Using the data-processing inequality, we then obtain

$$
\begin{aligned}
& \mathcal{R}\left(\rho^{Q} \otimes \rho^{R}, H^{Q R}\right) \\
& \quad=D_{2}\left(\omega_{H^{Q R}}\left(\rho^{Q} \otimes \rho^{R}\right) \| \mathbb{1} / d_{Q R}\right) \\
& \quad \geqslant D_{2}\left(\left(\omega_{H^{Q}} \otimes \omega_{H^{R}}\right) \circ \omega_{H^{Q R}}\left(\rho^{Q} \otimes \rho^{R}\right) \| \mathbb{1} / d_{Q R}\right) \\
& =D_{2}\left(\omega_{H^{Q}} \otimes \omega_{H^{R}}\left(\rho^{Q} \otimes \rho^{R}\right) \| \mathbb{1} / d_{Q R}\right) \\
& \quad=\mathcal{R}\left(\rho^{Q}, H^{Q}\right)+\mathcal{R}\left(\rho^{R}, H^{R}\right)
\end{aligned}
$$

which finishes the proof.

\section{Comparison of effective dimensions}

As explained in the main text, our results are based on the effective dimension as a suitable quantifier of the equilibrating properties of a system, which is justified by bounds (2) and (3). Nonetheless, there exists in the literature on the topic similar bounds formulated in terms of quantities other than effective dimension. That said, it seems natural to investigate whether alternative definitions of resilience can be put forward based on those other bounds, or even based on bounds that, although not yet shown to hold, are conceivably true.

First let us consider bounds of the form (2) and (3) based on the second largest of the eigenvalues of the time-averaged, dephased state [3]. This bound takes the form

$$
\operatorname{Var}_{H}(A, \rho) \leqslant\|A\|^{2} 3 p^{2^{\text {nd }}},
$$

where $p^{2^{\text {nd }}}$ is the second largest eigenvalue of $\omega_{H}(\rho)$. Indeed, in some situations this bound is stronger than the one based on the effective dimension. However, our bounds on the cost of preparing states that do not equilibrate cannot be analogously carried over using $p^{2^{\text {nd }}}$, instead of the effective dimension, since $p^{2^{\text {nd }}}$ is not a monotone under mixtures of unitaries [32]. That is, it is not possible to construct an alternative measure of resilience based on $p^{2^{\text {nd }}}$ that fulfills monotonicity and additivity as given by (14) and (15).

We now discuss, in light of our results, the possibility of strengthening bounds on equilibration. Note that combining Eqs. (2), (3), and (13), we see that

$$
\operatorname{Var}_{H}(A, \rho) \leqslant\|A\|^{2} e^{-S_{2}\left(\omega_{H}(\rho)\right)},
$$

where $S_{2}$ is the Rényi-2 entropy. Here, the Rényi- $\alpha$ entropy is defined as

$$
S_{\alpha}(\rho):=\log (d)-D_{\alpha}(\rho \| \mathbb{1} / d)=\frac{1}{1-\alpha} \log \left(\operatorname{Tr}\left(\rho^{\alpha}\right)\right) .
$$

Equation (79) strongly suggests considering the possibility of having bounds in terms of Rényi entropies $S_{\alpha}$ with $\alpha \neq 2$, i.e., bounds of the form

$$
\operatorname{Var}_{H}(A, \rho) \leqslant\|A\|^{2} g\left(S_{\alpha}\left(\omega_{H}(\rho)\right)\right),
$$

where $g$ is any function so that $\lim _{x \rightarrow \infty} g(x)=0$. Let us first consider the case $\alpha=1$, which yields the usual von Neumann entropy $S_{1}$. We show now that a bound of the form (80) for $\alpha=1$ is impossible, since the state constructed in Eq. (54) provides a counterexample. The energy distribution of this nonequilibrating state [see the discussion below Eq. (58)] is

$$
p_{1}=p_{2}=\frac{a}{2}, \forall 2<k \leqslant d: p_{k}=\frac{1-a}{d-2} .
$$

Using the entropy scaling given in Eq. (59), it follows that $S_{1}$ grows linearly with the system's size, although the system does not equilibrate. The implications of the impossibility of a bound of the type (80) with $\alpha=1$ in relation to Theorem 3 are laid out in the next section.

The previous counterexample together with the fact that $S_{\alpha}(\rho) \geqslant S_{\alpha^{\prime}}(\rho)$ if $\alpha \leqslant \alpha^{\prime}$ (see Ref. [29]) implies that it is also impossible to find bounds of the form (80) for $\alpha \leqslant 1$.

\section{E. Theorem 3 and its relation to superadditivity}

As mentioned above, our results also imply that meaningful equilibration bounds cannot be derived in terms of the von Neumann entropy of the time-averaged state. In this subsection, we explain in detail how this relates to Theorem 3. To do so, let us assume the contrary, that is, that a bound of the form (80) with $\alpha=1$ would exist. In this case, a sensible definition of resilience would be given by

$$
\tilde{\mathcal{R}}(\rho, H):=D_{1}\left(\omega_{H}(\rho) \| \mathbb{1} / d\right) .
$$

As can be easily seen from the properties of the quantum relative entropy, $\tilde{\mathcal{R}}$ is also additive and fulfills the data-processing inequality. Hence, Theorem 1 also holds in terms of $\tilde{\mathcal{R}}$. However, $\tilde{\mathcal{R}}$ has the additional property of being superadditive, meaning that if we consider a bipartite, noninteracting system in a possibly correlated state $\rho^{Q R}$, we have

$$
\tilde{\mathcal{R}}\left(\rho^{Q R}, H^{Q} \otimes \mathbb{1}+H^{R} \otimes \mathbb{1}\right) \geqslant \tilde{\mathcal{R}}\left(\rho^{Q}, H^{Q}\right)+\tilde{\mathcal{R}}\left(\rho^{R}, H^{R}\right) .
$$

This follows from the superadditivity of the quantum relative entropy (or equivalently from the subadditivity of the von Neumann entropy) [32]. We can use this property to obtain an even stronger result than Theorem 1 by essentially the same calculation:

$$
\begin{aligned}
& \tilde{\mathcal{R}}\left(\sigma^{Q}, H^{Q}\right)+\tilde{\mathcal{R}}\left(\sigma^{R}, H^{R}\right) \geqslant \tilde{\mathcal{R}}\left(\rho^{Q R}, H^{R}+H^{Q}\right) \\
& \quad \geqslant \tilde{\mathcal{R}}\left(\rho^{Q}, H^{Q}\right)+\tilde{\mathcal{R}}\left(\rho^{R}, H^{R}\right) .
\end{aligned}
$$

Rearranging, we obtain the second-law-like inequality

$$
-\Delta \tilde{\mathcal{R}}^{R} \geqslant \Delta \tilde{\mathcal{R}}^{Q} .
$$

Hence, one concludes that the results of Theorem 3, showing a violation of this second-law-like inequality, imply that it is not possible to find equilibration bounds of the form of Eqs. (2) and (3) in terms of a subadditive entropy, or equivalently a superadditive divergence. 


\section{CONCLUSIONS AND OUTLOOK}

Using an approach inspired by so-called resource theories [38-43], we have studied the cost of preparing states that do not equilibrate. We have shown that in order to prepare a large interacting system in a state that can withstand equilibration in the long run, it is necessary to have access to another large system that can withstand equilibration. We have also discussed readings of what could be called a second law of equilibration for dynamics under unitary evolutions, with an emphasis on the role of correlations. Our work connects the recently emerging field of resource theories of coherence [23-25,27,34,44-48] with the pure-state quantum statistical mechanics approach, establishing a bridge between two subfields of modern quantum thermodynamics. Our results show that such a resource theoretical approach can be used to derive general results about longstanding problems in the foundations of statistical mechanics via information theoretic methods.

A promising further research area related to the present one involves providing bounds to the resources needed to prepare states that do not thermalize. That is, there are efforts to provide bounds on the resources that are needed to prepare states that, although possibly reaching an equilibrium state, do not equilibrate to a canonical ensemble. Given that equilibration to a canonical ensemble is ubiquitous in many-body systemswith seminal exceptions such as systems exhibiting many-body localization - one might expect that the preparation of states in initial conditions that escape thermalization requires, similar to the case of systems avoiding equilibration, a large amount of resources. As mentioned before, it would also be interesting to incorporate additional natural physical constraints into our framework, such as energy conservation or limited unitary control. While such additional constraints could only lead to tighter bounds for the required resources to prepare a system that does not equilibrate, they could nevertheless rule out the possibility of preparing arbitrarily many systems that do not equilibrate using a single resource in the sense of Theorem 3. We leave these questions for future work.

\section{ACKNOWLEDGMENTS}

The group at FUB thanks the DFG (EI 519/7-1, GA 2184/21, CRC 183-project B02), the European Research Council (TAQ), and the EC (AQuS) for support. H.W. further thanks the Studienstiftung des Deutschen Volkes for support. C.G. is supported by a Marie Skodowska-Curie Individual Fellowships (IF-EF) program under GA: 700140 by the European Union and acknowledges financial support from the European Research Council (CoG QITBOX and AdG OSYRIS), the Axa Chair in Quantum Information Science, Spanish MINECO (FOQUS FIS2013-46768, QIBEQI FIS2016-80773-P, and Severo Ochoa Grant No. SEV-2015-0522), and the Fundació Privada Cellex, and Generalitat de Catalunya (Grant No. SGR 874 and 875, and the CERCA Program).
[1] P. Reimann, Phys. Rev. Lett. 101, 190403 (2008).

[2] N. Linden, S. Popescu, A. J. Short, and A. Winter, New J. Phys. 12, 055021 (2010).

[3] P. Reimann and M. Kastner, New J. Phys. 14, 043020 (2012).

[4] C. Gogolin and J. Eisert, Rep. Prog. Phys. 79, 056001 (2016).

[5] A. J. Short and T. C. Farrelly, New J. Phys. 14, 013063 (2012).

[6] J. Eisert, M. Friesdorf, and C. Gogolin, Nat. Phys. 11, 124 (2015).

[7] A. Polkovnikov, K. Sengupta, A. Silva, and M. Vengalattore, Rev. Mod. Phys. 83, 863 (2011).

[8] P. Reimann, Phys. Scr. 86, 58512 (2012).

[9] N. Linden, S. Popescu, A. J. Short, and A. Winter, Phys. Rev. E 79, 061103 (2009).

[10] A. S. L. Malabarba, L. P. García-Pintos, N. Linden, T. C. Farrelly, and A. J. Short, Phys. Rev. E 90, 012121 (2014).

[11] C. Gogolin, Phys. Rev. E 81, 051127 (2010).

[12] S. Goldstein, T. Hara, and H. Tasaki, Phys. Rev. Lett. 111, 140401 (2013).

[13] S. Goldstein, T. Hara, and H. Tasaki, New J. Phys. 17, 045002 (2015).

[14] L. P. García-Pintos, N. Linden, A. S. L. Malabarba, A. J. Short, and A. Winter, Phys. Rev. X 7, 031027 (2017).

[15] P. Reimann, Nat. Commun. 7, 10821 (2016).

[16] L. Masanes, A. J. Roncaglia, and A. Acín, Phys. Rev. E 87, 032137 (2013).

[17] H. Wilming, M. Goihl, C. Krumnow, and J. Eisert, arXiv:1704.06291.

[18] T. R. de Oliveira, C. Charalambous, D. Jonathan, M. Lewenstein, and A. Riera, New J. Phys. 20, 033032 (2018).

[19] J. Goold, M. Huber, A. Riera, L. del Rio, and P. Skrzypczyk, J. Phys. A 49, 143001 (2016).
[20] G. Gour, M. P. Müller, V. Narasimhachar, R. W. Spekkens, and N. Y. Halpern, Phys. Rep. 583, 1 (2015).

[21] M. Horodecki, P. Horodecki, and J. Oppenheim, Phys. Rev. A 67, 062104 (2003).

[22] J. Aberg, arXiv:quant-ph/0612146.

[23] T. Baumgratz, M. Cramer, and M. B. Plenio, Phys. Rev. Lett. 113, 140401 (2014).

[24] A. Winter and D. Yang, Phys. Rev. Lett. 116, 120404 (2016).

[25] A. Streltsov, G. Adesso, and M. B. Plenio, Rev. Mod. Phys. 89, 041003 (2017).

[26] I. Marvian and R. W. Spekkens, Phys. Rev. A 94, 052324 (2016).

[27] A. Streltsov, S. Rana, P. Boes, and J. Eisert, Phys. Rev. Lett. 119, 140402 (2017).

[28] E. H. Lieb and D. W. Robinson, Commun. Math. Phys. 28, 251 (1972).

[29] M. Tomamichel, Quantum Information Processing with Finite Resources, Mathematical Foundations, SpringerBriefs in Mathematical Physics, Vol. 5 (Springer, Cham, 2016).

[30] C. Tsallis, J. Stat. Phys. 52, 479 (1988).

[31] M. P. Mueller, arXiv:1707.03451.

[32] M. Nielsen and I. Chuang, Quantum Information and Computation (Cambridge University Press, Cambridge, 2000).

[33] H. Wilming, M. Goihl, I. Roth, and J. Eisert, arXiv:1802.02052.

[34] S. D. Bartlett, T. Rudolph, and R. W. Spekkens, Rev. Mod. Phys. 79, 555 (2007).

[35] M. Lostaglio, K. Korzekwa, and A. Milne, Phys. Rev. A 96, 032109 (2017).

[36] A. M. Alhambra, L. Masanes, J. Oppenheim, and C. Perry, Phys. Rev. X 6, 041017 (2016). 
[37] T. Farrelly, F. G. S. L. Brandão, and M. Cramer, Phys. Rev. Lett. 118, 140601 (2017).

[38] M. Horodecki, J. Oppenheim, and R. Horodecki, Phys. Rev. Lett. 89, 240403 (2002).

[39] F. G. S. L. Brandão and M. B. Plenio, Nat. Phys. 4, 873 (2008).

[40] F. G. S. L. Brandão, M. Horodecki, J. Oppenheim, J. M. Renes, and R. W. Spekkens, Phys. Rev. Lett. 111, 250404 (2013).

[41] F. G. S. L. Brandão and G. Gour, Phys. Rev. Lett. 115, 070503 (2015).
[42] L. del Rio, L. Kraemer, and R. Renner, arXiv:1511.08818.

[43] B. Coecke, T. Fritz, and R. W. Spekkens, Inf. Comput. 250, 59 (2016).

[44] J. A. Vaccaro, F. Anselmi, H. M. Wiseman, and K. Jacobs, Phys. Rev. A 77, 032114 (2008).

[45] G. Gour and R. W. Spekkens, New J. Phys. 10, 033023 (2008).

[46] I. Marvian and R. W. Spekkens, Phys. Rev. A 90, 062110 (2014).

[47] M. Lostaglio, D. Jennings, and T. Rudolph, Nat. Commun. 6, 6383 (2015).

[48] M. Lostaglio, K. Korzekwa, D. Jennings, and T. Rudolph, Phys. Rev. X 5, 021001 (2015). 